\title{
Turning biodefense dollars into products
}

\author{
Melanie C Trull, Tracey V du Laney \& Mark D Dibner
}

\begin{abstract}
Five years after the US anthrax attacks, and more than two years after BioShield legislation was ratified, a survey reveals that biodefense funding has thus far produced only a handful of products for clinical development.
\end{abstract}

W ith the signing of the Pandemic and All-Hazards Preparedness Act into law in December 2006, the Biomedical Advanced Research and Development Authority (BARDA) was created, and given a \$1.07-billion budget for the next two years to galvanize the development of new products to counter security threats, including biological threats. Although it is hoped this will push more products from preclinical research through the clinic, a survey of R\&D programs on biodefense reveals that only a very small number of vaccines and therapeutics targeting potential bioterrorism agents are inching their way into the clinic.

\section{A survey of biodefense}

Biowarfare and bioterrorism have been used for centuries to terrorize, weaken, incapacitate and defeat armies and whole populations of people. Historically, the pathogens used were naturally occurring, such as the plague and smallpox. However, with new genetic manipulation technologies and bacterial and viral culturing capabilities, it is possible to engineer organisms with resistance to common antibiotics and antivirals for conducting bioterrorism attacks.

In addition, with advancements in modern travel and trade, diseases endemic to specific geographic regions can be spread across the globe. For example, severe acute respiratory syndrome (SARS) spread from Southeast Asia throughout the world within a matter of weeks in 2003 through international travel. The resulting worldwide disruptions in international business, travel and daily life were widely reported in the media and created fear in many members of the public. Regional or local dis-

BioAbility, LLC, PO Box 14569, Research Triangle Park, North Carolina 27709, USA. e-mail: mtrull@bioability.com eases were once regarded as 'traveler's diseases' that had little probability of spreading through developed countries with advanced public health systems. However, as the SARS epidemic showed, even cities with advanced hospital and public health systems, such as Hong Kong and Toronto, could not initially contain and control the spread of the disease.

To present a clear picture to those interested in biodefense disease prevention and treatment, we have accessed commercial drug pipeline databases, government publications and websites, company websites and pharmaceutical news reporting services to identify therapeutics and vaccines in development, to sort through inconsistencies and omissions and to determine the stage of development for these products. The US Centers for Disease Control and Prevention (CDC)'s list of bioterrorism agents (Box 1) served to define the agents we studied. We searched for products in development for all Category A and Category B agents, as well as for four Category $\mathrm{C}$ agents: CrimeanCongo hemorrhagic fever virus, yellow fever, avian influenza (as a subset of influenza) and SARS-associated corona virus (SARS-CoV). These four were selected as the most likely to have products in development that are biodefense driven. Further details of this study and the basis for all data shown in this article are available elsewhere ${ }^{1}$.

The US CDC list remains the government's best public estimate of current biological threats and provides direction for research and product development. However, the list is an active document and should be expected to change over time, as new threats emerge and vaccines or therapeutics are developed

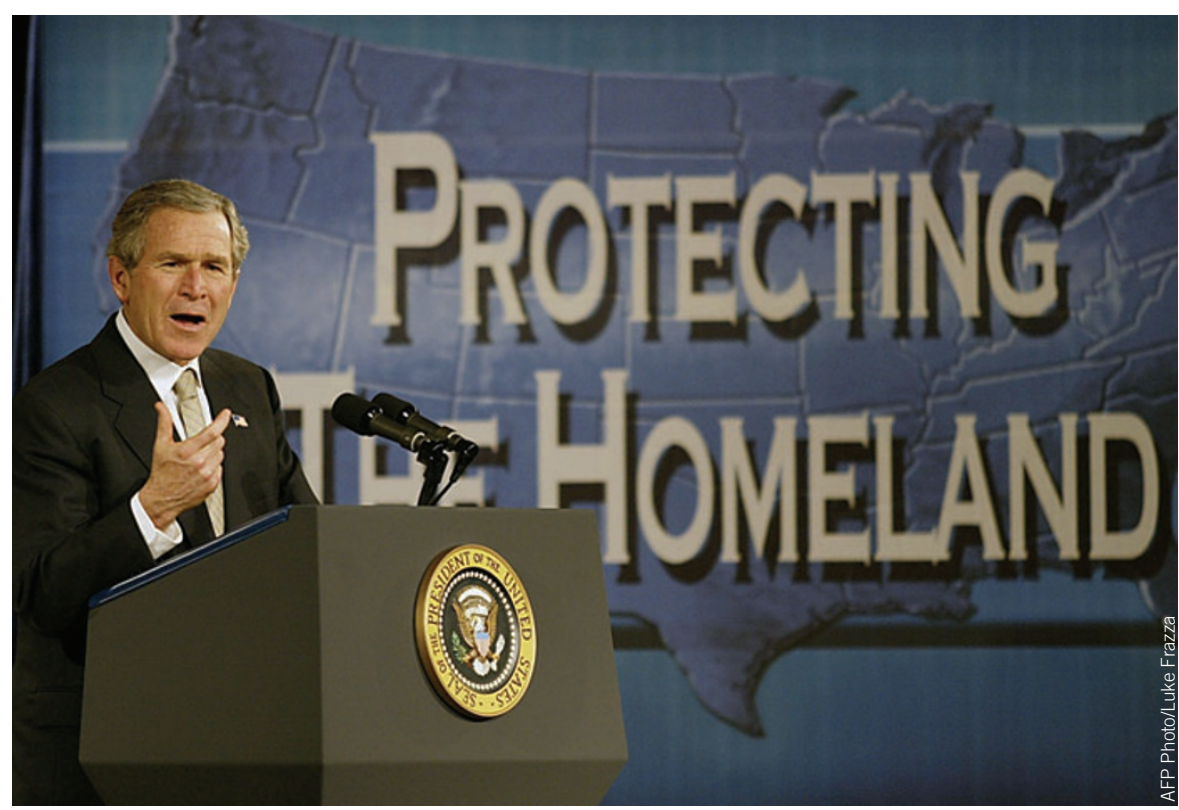

President Bush encourages the US Congress to pass BioShield legislation. 


\section{Box 1 Potential bioterrorism diseases and agents}

The CDC classifies agents that could be used in bioterrorism into three categories: category A, B or C (for details, see ref. 10).

Category A agents. These nine agents or families of agents are the bioterrorism agents of highest priority for biodefense research. The CDC defines Category A agents as organisms that pose a risk to national security because they are easily disseminated or transmitted from person to person, result in high mortality rates and have the potential for a major public health impact, might cause public panic and social disruption, and require special action for public health preparedness.

- Anthrax (Bacillus anthracis)

- Botulism (Clostridium botulinum toxin)

- Plague (Yersinia pestis)

- Smallpox (Variola major)

- Tularemia (Francisella tularensis)

Viral hemorrhagic fevers

- Arenaviruses. Lymphocytic choriomeningitis virus, Junin virus, Machupo virus, Guanarito virus, Lassa fever

- Bunyaviruses. Hantaviruses, Rift Valley fever

- Flaviviruses. Dengue

- Filoviruses. Ebola, Marburg

Category B agents. The CDC defines Category B agents as organisms that are moderately easy to disseminate, result in moderate morbidity rates and low mortality rates, and require specific enhancements of CDC's diagnostic capacity and enhanced disease surveillance.

- Burkholderia pseudomallei

- Coxiella burnetii (Q fever)

- Brucella spp. (brucellosis)

- Burkholderia mallei (glanders)

- Ricin toxin (from Ricinus communis)
- Epsilon toxin of Clostridium perfringens

- Staphylococcus enterotoxin B

- Typhus fever (Rickettsia prowazekii)

Food and waterborne pathogens

- Bacteria. Diarrheagenic Escherichia coli, pathogenic Vibrio spp., Shigella spp., Salmonella spp., Listeria monocytogenes, Campylobacter jejuni and Yersinia enterocolitica

- Viruses. Caliciviruses, hepatitis A

- Protozoa. Cryptosporidium parvum, Cyclospora cayatanensis, Giardia lamblia, Entamoeba histolytica, Toxoplasma and microsporidia

Additional encephalitide viruses

- West Nile, La Crosse, California encephalitis, Venezuelan equine encephalitis, Eastern equine encephalitis, Western equine encephalitis, Japanese encephalitis and Kyasanur Forest

Category $\mathbf{C}$ agents. These include emerging pathogens that could be engineered for mass dissemination in the future because of availability, ease of production and dissemination, and potential for high morbidity and mortality rates and major health impact.

- Tick-borne hemorrhagic fever viruses

- Crimean-Congo hemorrhagic fever virus

- Tick-borne encephalitis viruses

- Yellow fever

- Multidrug-resistant tuberculosis

- Influenza

- Other Rickettsias

- Rabies

- Severe acute respiratory syndrome-associated coronavirus (SARS-CoV) for existing threats. For example, companies are developing vaccines and therapeutics to target existing pathogens, but terrorists may genetically modify existing agents or create new agents that are serious threats.

Even when an effective vaccine is available against a possible bioterrorism disease, the agent may remain a threat because the vaccine is not routinely administered. Thus, if the United States resumed universal administration of a smallpox vaccine, smallpox would no longer be considered a top bioterrorism threat. The existing smallpox vaccines, however, can cause serious complications. This issue limits the utility of these vaccines and is driving the development of safer smallpox vaccines.

\section{US government spending initiatives}

In July 2004, BioShield legislation was signed that would provide $\$ 5.6$ billion over ten years to purchase vaccines and therapeutics for bioterrorism agents. Although this legislation creates a guaranteed market, it was designed for endstage acquisition, not to fund development. The first and largest contract awarded under
BioShield was announced in November 2004. Worth $\$ 877.5$ million over a five-year period, it was awarded to VaxGen (Brisbane, CA, USA) to manufacture and deliver 75 million doses of anthrax vaccine for the Strategic National Stockpile. The contract was contentious, with product delays and contract modifications; ultimately, the US Department of Health and Human Services terminated the contract in December 2006 (ref. 2).

It is still unclear how successful BioShield will be in promoting development of biodefense products. To date, few large pharmaceutical companies have been attracted to the biodefense market by the promise of BioShield funding. The amount of money involved is not great enough to offset concerns about liability and limited market potential. Although a second bill, BioShield II, was introduced in April 2005, it has still not been approved. BioShield II authorizations include tax incentives to spur capital investment, intellectual property protection and liability limits.

More than half of the funding we identified for the development of vaccines and therapeutics for bioterrorism agents was targeted to anthrax. However, the priority for government spending on biodefense products is not always evident. In the fall of 2005, there was a large infusion of funding from the National Institute of Allergy and Infectious Disease (NIAID) for tularemia, which is usually treatable with antibiotics. Cerus (Concord, CA, USA) and the University of New Mexico (Albuquerque, NM, USA) received a \$23 million grant to research and develop a tularemia vaccine, whereas DynaPort Vaccine Company (DVC; Frederick, MD, USA) was awarded a fiveyear \$35.1 million contract for the development of a tularemia vaccine. The National Biodefense Analysis and Countermeasures Center has completed an assessment and ranking of the bioterrorism threats, which is expected to guide federal spending. Although that assessment was delivered to the White House in January 2006, it has been seen only by a small group of individuals with high-level security clearance ${ }^{3}$.

The creation of BARDA provides additional product development funding. BARDA's budget was authorized to facilitate the development 
of new products to counter security threats, including biological threats. This is expected to bridge the funding gap between initial research and end product that is particularly challenging for smaller companies.

\section{Vaccines in development}

We identified 152 prophylactic vaccines in development as well as six recently launched vaccines against bioterrorism threats (Table 1). Among vaccines for Category A agents, development is concentrated on anthrax, with 24 vaccines in development, followed by 19 for smallpox and 13 for plague. There is also significant activity in other categories, with 14 vaccines in development for viral encephalitides and 28 vaccines in development for avian influenza ${ }^{1}$.

Of these 152 vaccines, 102 are in preclinical development, 35 are in phase 1, 12 are in phase 2 , and only three are in phase 3 clinical trials (Fig. 1). The pipeline is dominated by products in early stages of development, with few vaccines likely to reach the market in the next few years $^{1}$ (Table 2 ).

There are a number of challenges to creating biodefense vaccines, including providing protection against multiple serotypes. Many of the pathogens have unstable genomes, multiple genotypes or multiple serotypes. As one example, there are seven known serotypes of the Clostridium botulinum neurotoxin that causes botulism. To be completely effective, a vaccine must protect against all seven serotypes of neurotoxin. There is one vaccine for botulism that is in phase 1 trials, but it is only effective against two of the serotypes. The remaining five vaccines are in preclinical development, and only one of those is heptavalent. A heptavalent vaccine that is cost effective may find acceptance outside the biodefense market. An additional constraint is the recognition that vaccines created for the Strategic National Stockpile must have other attributes, such as stability, ability to be manufactured on a large scale and high-volume administration.

For some agents on the list, such as hepatitis $\mathrm{A}$, an effective vaccine already exists. In fact, we have identified more than a dozen hepatitis A vaccines on the market worldwide, with three approved in the United States. Hepatitis A, however, is still a bioterrorism concern, because the vaccine is not routinely administered. In 2004, the CDC estimated there were 56,000 new infections in the United States, but only 5,683 cases were reported to the National Notifiable Disease Surveillance System. According to the CDC, hepatitis A vaccines were more widely used in the late 1990s, and the number of cases reached historic lows 4 . Although vaccines have already been approved for several bioterrorism agents, some companies are developing vaccines with greater efficacy, improved safety or easier administration. For example, Hadassah Medical Organization (Jerusalem) has an edible vaccine for hepatitis A in preclinical development.

One substantial change since we surveyed the field in 2005 (ref. 5) is a decrease in the number of vaccines in development for SARS. The SARS-associated coronavirus was added as a Category C agent in August 2004, after an outbreak in 2002-2003 that infected more than 8,000 people. In 2005 , we identified 18 vaccines in development, but by 2006 this number had dropped to ten ${ }^{5}$. Active development of SARS products has been delayed or suspended at many companies, because the disease has not

\section{Table 1 Summary of biodefense products in development and their sponsors}

\begin{tabular}{|c|c|c|c|c|}
\hline Bioterror disease/agent & Total biodefense vaccines & $\begin{array}{l}\text { Total firms developing } \\
\text { biodefense vaccines }\end{array}$ & Total biodefense therapeutics & $\begin{array}{c}\text { Total firms developing } \\
\text { biodefense therapeutics }\end{array}$ \\
\hline \multicolumn{5}{|l|}{ Category A } \\
\hline Anthrax (Bacillus anthracis) & 24 & 21 & 17 & 21 \\
\hline Botulism (Clostridium botulinum) & 6 & 6 & 5 & 6 \\
\hline Plague (Yersinia pestis) & 13 & 16 & 8 & 7 \\
\hline Smallpox virus (Variola major) & 19 & 18 & 20 & 19 \\
\hline Tularemia (Francisella tularensis) & 4 & 5 & 0 & 0 \\
\hline Arenaviruses & 1 & 1 & 6 & 5 \\
\hline Hantaviruses and Rift Valley fever & 1 & 1 & 2 & 2 \\
\hline Dengue virus & 9 & 9 & 10 & 11 \\
\hline Ebola and Marburg viruses & 6 & 6 & 12 & 13 \\
\hline \multicolumn{5}{|l|}{ Category B } \\
\hline Burkholderia pseudomallei & 0 & 0 & 1 & 1 \\
\hline Q fever (Coxiella burnetii) & 0 & 0 & 1 & 1 \\
\hline Brucellosis (Bruceila spp.) & 0 & 0 & 2 & 2 \\
\hline Ricin toxin (from Ricinus communis) & 2 & 2 & 3 & 4 \\
\hline Epsilon toxin of Clostridium perfringens & 0 & 0 & 0 & 0 \\
\hline Staphylococcus enterotoxin B & 2 & 2 & 1 & 2 \\
\hline Typhus fever (Rickettsia prowazekii) & 2 & 2 & 0 & 0 \\
\hline Food and waterborne bacteria & 12 & 11 & 6 & 6 \\
\hline Food and waterborne viruses & 3 & 3 & 1 & 1 \\
\hline Food and waterborne protozoa & 0 & 0 & 9 & 7 \\
\hline Viral encephalitide viruses & 14 & 15 & 18 & 16 \\
\hline \multicolumn{5}{|l|}{ Category C } \\
\hline Crimean-Congo hemorrhagic fever virus & 0 & 0 & 1 & 1 \\
\hline Yellow fever & 2 & 2 & 2 & 2 \\
\hline SARS & 10 & 10 & 14 & 16 \\
\hline Avian influenza & 28 & 27 & 16 & 16 \\
\hline
\end{tabular}

Source: ref. 1. 


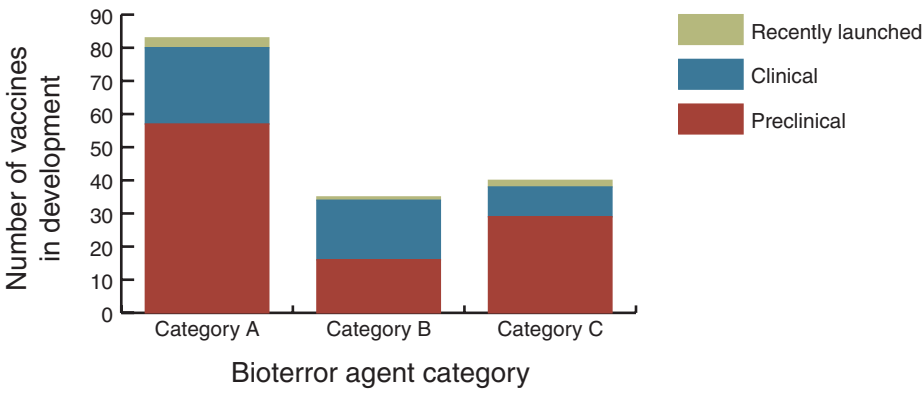

Figure 1 Biodefense vaccines in development.

been a major health concern since the 2003 outbreak. Berna Biotech (Bern, Switzerland), which had a vaccine in preclinical development, announced that it was abandoning clinical trials of its vaccine because the disease was no longer seen as a priority by global health authorities. Other companies have indicated that the market and epidemiological developments must be evaluated before investing further resources into the development of vaccines or therapeutics for SARS. However, SARS-CoV remains in animal reservoirs and may reemerge.

\section{Therapeutics in development}

In terms of therapeutics, 155 products are in development or recently launched against bioterrorism diseases (Table 1). Among therapeutics for Category A and B agents, development is concentrated on smallpox, with 20 therapeutics in development, followed by 18 for viral encephalitides and 17 for anthrax. Except for therapeutics targeting encephalitide viruses, few products are in development for Category B agents. There is significant activity against two Category $\mathrm{C}$ agents, however, with 14 and 16 therapeutics in development for SARS and avian influenza, respectively ${ }^{1}$. Of these 155 therapeutics, 129 are in preclinical development, with 16 in clinical trials and 10 recently launched (Fig. 2). As for vaccines, the therapeutic pipeline is dominated by products in preclinical development ${ }^{1}$.

For those working on therapeutics against bioterrorism diseases, the goal is to develop products capable of treating a variety of infections. At the outset, many of these diseases present with 'flu-like' symptoms and broadspectrum therapeutics would be of the greatest utility so that treatment can be initiated before identification of the causative agent. Although numerous broad-spectrum antibiotics are already available, few have been tested and approved for the treatment of specific bioterrorism infections.

Products that are currently used as the standard-of-care include ciprofloxacin (Cipro; Bayer Healthcare, Wuppertal, Germany) for the treatment of anthrax infections and vaccinia immune globulins for the treatment of side effects of smallpox vaccine administration. Development of therapeutics for viral

\section{Table 2 Biodefense vaccines and therapeutics in phase 2 or phase 3 clinical trials}

\begin{tabular}{|c|c|c|c|c|}
\hline Bioterror agent & Company (location) & Type of product & Name & Stage \\
\hline Anthrax & VaxGen & Vaccine & rPA102 anthrax vaccine & Phase 2 \\
\hline Plague & $\begin{array}{l}\text { Defence Science and Technology } \\
\text { Laboratories (Porton Down, UK) }\end{array}$ & Vaccine & $\begin{array}{l}\text { F1/N combination recombinant subunit } \\
\text { plague vaccine }\end{array}$ & Phase 2 \\
\hline Smallpox & Acambis & Vaccine & ACAM2000 & Phase 3 \\
\hline Smallpox & Acambis & Vaccine & MVA3000 & Phase 2 \\
\hline Smallpox & $\begin{array}{l}\text { Bavarian Nordic } \\
\text { (Kvistgard, Denmark) }\end{array}$ & Vaccine & IMVAMUNE & Phase 2 \\
\hline Dengue & Acambis/Sanofi Pasteur & Vaccine & ChimeriVax-Dengue & Phase 2 \\
\hline Dengue & $\begin{array}{l}\text { GlaxoSmithKline Biologicals } \\
\text { (Rixensart, Belgium) }\end{array}$ & Vaccine & Attenuated tetravalent dengue vaccine & Phase 2 \\
\hline Dengue & Sanofi Pasteur & Vaccine & Attenuated tetravalent dengue vaccine & Phase 2 \\
\hline Food and waterborne bacteria & $\begin{array}{l}\text { AVANT Immunotherapeutics } \\
\text { (Needham, MA, USA) }\end{array}$ & Vaccine & CholeraGarde & Phase 2 \\
\hline Food and waterborne bacteria & $\begin{array}{l}\text { Emergent Europe } \\
\text { (Wokingham, UK) }\end{array}$ & Vaccine & Oral typhoid vaccine & Phase 2 \\
\hline Food and waterborne bacteria & $\begin{array}{l}\text { Teijin Pharma } \\
\text { (Tokyo) }\end{array}$ & Therapeutic & Humanized $\mathrm{mAb}$ & Phase 2 \\
\hline Food and waterborne bacteria & AVANT Immunotherapeutics & Vaccine & Ty800 typhoid vaccine & Phase $1 / 2$ \\
\hline Food and waterborne bacteria & $\begin{array}{l}\text { lomai Corporation } \\
\text { (Gaithersburg, MD, USA) }\end{array}$ & Vaccine & ETEC vaccine patch & Phase $1 / 2$ \\
\hline Food and waterborne protozoa & GlaxoSmithKline & Therapeutic & Albendazole & Phase 3 \\
\hline Viral encephalitides & Acambis & Vaccine & ChimeriVax-JE & Phase 3 \\
\hline Viral encephalitides & $\begin{array}{l}\text { Intercell } \\
\text { (Vienna) }\end{array}$ & Vaccine & Japanese encephalitis vaccine & Phase 3 \\
\hline Viral encephalitides & Acambis & Vaccine & ChimeriVax-West Nile & Phase 2 \\
\hline Viral encephalitides & $\begin{array}{l}\text { Hemispherx Biopharma } \\
\text { (Philadelphia) }\end{array}$ & Therapeutic & Interferon-alpha-n3 & Phase 2 \\
\hline Viral encephalitides & AVI BioPharma & Therapeutic & AVI-4020 & Phase $1 \mathrm{~b} / 2$ \\
\hline Viral encephalitides & $\begin{array}{l}\text { OMRIX Biopharmaceuticals } \\
\text { (New York) }\end{array}$ & Therapeutic & Omr-IgG-am & Phase $1 / 2$ \\
\hline
\end{tabular}

Source: ref. 1. 


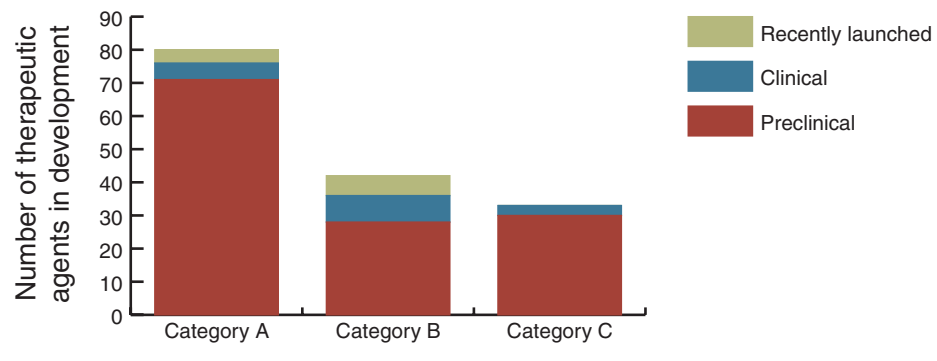

Bioterror agent category

Figure 2 Biodefense therapeutics in development.

diseases lags behind that for bacterial diseases, with most recommended care being palliative and nonspecific, such as the administration of liquids, fever reducers (e.g., ibuprofen and acetaminophen) and bed rest.

Many of the therapeutics in development are considered to be therapeutic vaccines. Therapeutic vaccines are often immune-modulating, agent-specific pharmaceuticals given after exposure, which protect the recipient from contracting the disease or diminish the severity of the disease. For example, researchers at the US Army Medical Research Institute of Infectious Diseases (USAMRIID, Fort Detrick, MD, USA) have developed two experimental therapeutic vaccines, which when given after exposure were capable of preventing Ebola and Marburg symptoms and lethality in guinea pigs and rhesus monkeys respectively ${ }^{6,7}$. Juvaris BioTherapeutics (Pleasanton, CA, USA) is also developing or codeveloping several therapeutic vaccines against potential bioterrorism agents, such as anthrax, smallpox and the plague.

\section{Companies and institutions developing biodefense products}

Of the companies, institutions or government entities with some program focused on biodefense, we identified a total of 94 that are developing vaccines and 95 developing therapeutics (Table 3). Groups developing vaccines were identified in 19 different countries and groups developing therapeutics were identified in 14 different countries. Companies in the United States dominate both lists, representing 61\% of the total in vaccine development and 64\% of the total in therapeutic development ${ }^{1}$. This result is partially due to the large number of pharmaceutical and biotech companies in the United States, but also partially from the availability of sources in the United States to identify and confirm companies involved in biodefense product development. Of the total, three government organizations and eight companies are developing both vaccines and therapeutics.

A broad range of companies is represented, including vaccine-focused multinationals
Sanofi Pasteur (Lyon, France) and Crucell (Leiden, The Netherlands). There are also companies focused on biodefense, such as DVC (Frederick, MD, USA), and small biotech firms such as Vaxin (Birmingham, AL, USA). Several government agencies in the United States, especially NIAID and USAMRIID, are involved. These agencies not only provide funding, but frequently have an active role in the development of biodefense vaccines.

Some companies are leveraging a proprietary technology to develop multiple products, each for a specific disease target. Acambis (Cambridge, UK) is developing vaccines for West Nile virus, Japanese encephalitis virus and dengue fever using its proprietary ChimeriVax technology, which is based on a live, attenuated
17D strain of the yellow fever virus (the basis for existing yellow fever vaccines) in which the genes encoding antigens conferring immunity to yellow fever virus have been replaced with genes from the virus of interest.

AlphaVax (Research Triangle Park, NC, USA) is developing vaccines against botulinum toxin, smallpox, SARS, Marburg virus and viral encephalitis using a proprietary vaccine vector system. The Alphavaccine Platform System is based on a nonpropagating alphavirus vector engineered to express selected genes from pathogens. Alphavaccine particles that contain the foreign antigen target the lymph nodes and infect the dendritic cells, initiating a strong immune response. Funding for AlphaVax's biodefense vaccine program exceeds $\$ 23$ million, coming primarily from the US federal government ${ }^{8}$.

Biotech company AVI BioPharma (Portland, OR, USA) is using its NeuGene antisense technology to develop therapeutics for a variety of biodefense diseases, including anthrax, dengue fever and Ebola. NeuGene antisense compounds have a synthetic chemical backbone designed to provide stability, specificity, efficacy, functional delivery and safety. The technology is expected to enable a rapid response to emerging threats. In December 2006, AVI BioPharma signed a two-year \$28 million contract with the Defense Threat Reduction Agency (Fort Belvoir, VA, USA) that will fund the development of

\begin{tabular}{|c|c|c|}
\hline Country & $\begin{array}{l}\text { Number of firms developing } \\
\text { biodefense vaccines }\end{array}$ & $\begin{array}{l}\text { Number of firms developing } \\
\text { biodefense therapeutics }\end{array}$ \\
\hline Argentina & 0 & 1 \\
\hline Australia & 2 & 4 \\
\hline Austria & 1 & 0 \\
\hline Belgium & 2 & 2 \\
\hline Canada & 3 & 6 \\
\hline China & 1 & 2 \\
\hline Denmark & 2 & 1 \\
\hline France & 2 & 1 \\
\hline India & 3 & 0 \\
\hline Israel & 2 & 3 \\
\hline Japan & 2 & 5 \\
\hline The Netherlands & 3 & 2 \\
\hline Russia & 1 & 0 \\
\hline Singapore & 1 & 2 \\
\hline South Korea & 2 & 0 \\
\hline Spain & 1 & 0 \\
\hline Sweden & 1 & 0 \\
\hline Switzerland & 1 & 0 \\
\hline Taiwan & 0 & 1 \\
\hline United Kingdom & 7 & 4 \\
\hline United States & 57 & 61 \\
\hline Total & 94 & 95 \\
\hline
\end{tabular}

Source: ref. 1. 
antisense therapeutics to treat Ebola, Marburg and Junin hemorrhagic viruses. This funding is in addition to an $\$ 11$ million allocation for AVI BioPharma's biodefense program as part of the 2006 Defense Appropriations Act ${ }^{9}$.

\section{Conclusions}

Despite many products currently in development, the outlook for biodefense vaccines and therapeutics is a bleak one, with few products on the market to counter most bioterrorism agents and only a few in late stages of development. Many companies, institutions and government agencies are working to reverse this situation. Even so, the rapidly changing landscape of public health priorities in infectious disease and necessary product attributes, such as high-volume administration, make biodefense a challenging area for development.

The primary market drivers for development of vaccines and therapeutic agents against diseases on the CDC list of bioterror agents are the continued threat posed by domestic and international terrorists and the availability of government funding. Some bioterrorism agents are an ongoing threat in the developing world and are also threats to travelers. In this category are diseases, such as yellow fever, dengue fever, Japanese encephalitis and typhoid fever. Several vaccines in development, targeted to travelers, have been designed to protect against a combination of food or waterborne agents.
The development of broad-spectrum antibiotics and antivirals that are tested and approved for use against bioterror diseases remains a pressing need. These diseases are often diseases endemic to developing countries and are not generally recognized in developed countries. Also, many produce generalized 'flu-like' symptoms at the outset and may not be recognized as bioterrorism related until a large number of infected individuals are identified and tracked. The availability of broad-spectrum antibiotics and antivirals that could be administered before identification of the etiological agent would be beneficial to the general medical community. Many of the broad-spectrum antibiotics already approved and marketed have never been tested against the diseases on the CDC list of potential bioterror agents and efficacy is not assured. The incentives for the large pharmaceutical companies to test their products against the bioterror diseases are minimal, involving large, protracted clinical trials in remote locations for diseases without large patient populations. The larger pharmaceutical companies may be leery of liability issues and the negative impact of failed products on the company's stock or venture capital investments.

Issues of delivery and stockpiling by federal, state and local governments also remain. Without guaranteed purchase by these large agencies, companies have little chance of recovering expenditures spent on the development of vaccines or therapeutics for most biodefense applications, and thus have little incentive to pursue them. Smaller pharmaceutical and biotech companies and institutions may have the compounds or technologies of utility in countering bioterrorism agents, but may not have the resources to complete product development and conduct clinical trials without additional support. BARDA may address some of these concerns, but it is too early to determine whether it will be effective.

1. Trull, M.C., du Laney, T.V. \& Dibner, M.D. Biodefense market report: vaccines, therapeutics, and diagnostics for bioterror agents 2006. BioAbility/BioWorld Atlanta, GA (June 2006).

2. HHS cancels VaxGen's anthrax vaccine contract. (VaxGen, Brisbane, CA, December 19, 2006). http:// biz.yahoo.com/prnews/061219/sftu092.html?.v=78

3. Warrick, J. The secretive fight against bioterror. The Washington Post (July 30, 2006) pA01.

4. http://www.cdc.gov/ncidod/diseases/hepatitis/a/fact. htm

5. Trull, M.C., du Laney, T.V. \& Dibner, M.D. Biodefense market report: drugs and vaccines for bioterror agents. BioAbility/BioWorld Atlanta, GA (May 2005).

6. Geisbert, T.W. et al. Postexposure protection of guinea pigs against a lethal Ebola virus challenge is conferred by RNA interference. J. Infect. Dis. 193, 1650-1657 (2006).

7. Daddario-DiCaprio, K.M. et al. Postexposure protection against Marburg haemorrhagic fever with recombinant vesicular stomatitis virus vectors in non-human primates: an efficacy assessment. Lancet 367, 13991404 (2006).

8. http://www.alphavax.com/products/bio.aspx

9. AVI BioPharma awarded $\$ 28$ million biodefense research contract by Department of Defense/DTRA. (AVI BioPharma, Portland, OR, December 4, 2006). http://www.avibio.com/pr/pr327.html

10. http://www.bt.cdc.gov/agent/agentlist-category.asp 